\title{
Investigating gamma-ray emission of the Cygnus cocoon with 12 years of Fermi-LAT data
}

\section{Xan Astiasarain, ${ }^{a, *}$ Luigi Tibaldo, ${ }^{a}$ Pierrick Martin $^{a}$ and Jürgen Knödlseder ${ }^{a}$ on behalf of the Fermi-LAT Collaboration \\ ${ }^{a}$ Institut de Recherche en Astrophysique et Planétologie, Université de Toulouse, CNRS, CNES, UPS, 9 avenue Colonel Roche, 31028 Toulouse, Cedex 4, France}

E-mail: xan.astiasarain@irap.omp.eu

\begin{abstract}
Massive star-forming regions are genuine reservoirs of energy and potential sources of nonthermal particles, but their role in Galactic cosmic-ray acceleration and propagation is still poorly understood. Gamma-ray observations of the Cygnus X star-forming region revealed the presence of a cocoon of freshly-accelerated cosmic rays, making it one of the best examples to investigate these issues. However, the exact acceleration sites and mechanisms, the nature of the particles, and how they propagate through the cocoon have not been firmly established yet, and the contribution from other gamma-ray sources in this crowded region remains uncertain. We present a new in-depth analysis of more than 12 years of observations from the Fermi Large Area Telescope combined with high-resolution interstellar medium tracer data to improve the understanding of gamma-ray emission from the Cygnus region. The evolution of the cocoon morphology as a function of energy and its spectrum up to $1 \mathrm{TeV}$ will be used to shed new light on the open questions about the physical processes shaping the young cosmic-ray population in Cygnus X.
\end{abstract}

$37^{\text {th }}$ International Cosmic Ray Conference (ICRC 2021)

July 12 th - 23rd, 2021

Online - Berlin, Germany

\footnotetext{
${ }^{*}$ Presenter
} 


\section{Introduction}

More than a century after the discovery of Galactic cosmic rays (CRs), their origin is still puzzling. Supernova remnants (SNR) are the principal candidates for sources of Galactic CRs via diffusive shock acceleration. However, the isotopic abundances in CRs indicate that $20 \%$ of the accelerated material comes from Wolf-Rayet stars, present in massive star-forming regions (MSFRs) [1]. Moreover MSFRs are large reservoirs of energy due to a high rate of supernovae and to the collective effect of massive-star winds. Therefore MSFRs are robust candidates for CR sources in alternative to SNRs.

The interactions of CRs produce $\gamma$ rays by interactions with interstellar gas (Bremsstrahlung and inelastic nucleon-nucleon collisions) and by inverse Compton scattering of CR electrons off low-energy photons. Hadronic $\gamma$ rays trace densities and interstellar gas densities in remote environments. Therefore, if we know the distribution of the interstellar gas toward a MSFR, we can look at interstellar $\gamma$-ray emission to determine its content.

In this work, we are focusing of the Cygnus $\mathrm{X}$ region, located in the local arm at approximately $1.4 \mathrm{kpc}$ from the solar system. A study of the Cygnus X region has already unveiled a cocoon of freshly accelerated [2] and more recently the Cygnus cocoon has been observed using HAWC data above $1 \mathrm{TeV}$ [3]. Other studies show the existence of potential $\gamma$-ray signals toward some MSFRs ([4], [5], [6], [7], [8], [9]), but not others ([10], [11]). The nature of the Cygnus cocoon, as well as of the gamma-ray emission from other MSFRs, and the particle acceleration and propagation mechanisms at play in MSFRs are still poorly understood. Therefore, we revisit gamma-ray emission from the Cygnus cocoon using 12 years of Fermi LAT data.

\section{Gas column-density maps}

We used different tracers to build maps of interstellar gas in its different phases along the line of sight.

In order to trace neutral atomic gas, we used the $21-\mathrm{cm}$ line from the hyperfine transition of atomic hydrogen $(\mathrm{HI})$. We used the radio survey from Effelsberg and Parkes observations called HI4PI [12] combined with the Canadian Galactic Plane Survey (CGPS, [13]) for the innermost part of the Cygnus region. We used a spin temperature of $250 \mathrm{~K}$ for the calculation of $\mathrm{H}$ i column densities. [14].

Molecular hydrogen cannot be directly traced. The tracer used here is the ${ }^{12} \mathrm{CO} J_{1 \rightarrow 0}$ rotational line that we observe at a wavelength of $2.6 \mathrm{~mm}$. We used data from the survey by the Centre for Astrophysics (CfA) [15]. We assumed that the $\mathrm{H}_{2}$ column densities are proportional to the integrated intensities of the 2.6-mm line, $W_{C O}$ with a conversion factor $X_{C O}$ :

$$
N\left(H_{2}\right)=X_{C O} \cdot W_{C O}
$$

From these data we built $\mathrm{H}$ i column density and $\mathrm{CO}$ emission intensity maps for the Cygnus $\mathrm{X}$ region. We separated the gas into three coherent structures along the line of sight in the longitude, latitude and Doppler shift velocity space. They correspond to the major Galactic arms: Local Arm, Perseus Arm, and Outer arm and beyond. We performed the separation using a decomposition of 


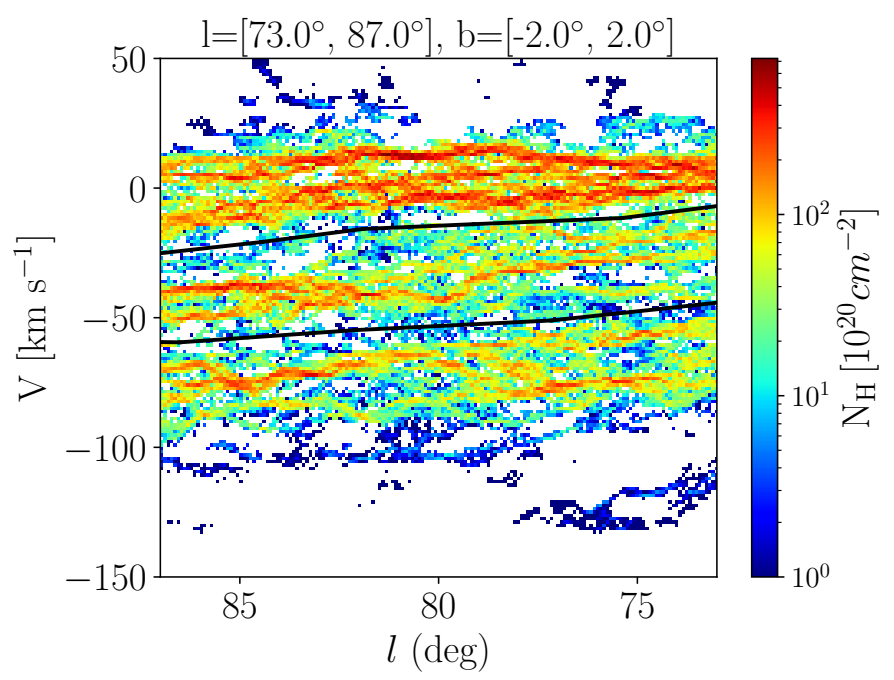

Figure 1: H i column density as a function of Doppler-shift velocity and Galactic longitude. Total column densities from each pseudo-Voigt profile were assigned to the peak position in velocity. The black lines show the boundaries that we defined o separate the three structures along the line of sight (from top to bottom): local arm, Perseus arm, and Outer Arm and beyond

the brightness temperature as a function of the Doppler velocity into pseudo-Voigt profiles [16] based on velocity boundaries inferred from longitude-velocity and latitude-velocity diagrams.

A significant fraction of interstellar gas is not traced neither by the $21-\mathrm{cm}$ line, nor by the CO $J_{1 \rightarrow 2}$ and is called the Dark Neutral Medium (DNM). The DNM is mainly composed of the gas at the transition of the atomic and molecular clouds, where we can find $\mathrm{H}_{2}$ without $\mathrm{CO}$ that is more easily photo-dissociated and optically thick H I [17].

Under the hypothesis that dust thermal emission is approximately proportional to gas column density along the line of sight, the DNM distribution can be obtained from the residuals on top of the components traced by $\mathrm{H}$ i and $\mathrm{CO}$. We do so using the dust opacity map at $353 \mathrm{GHz}$ obtained from Planck data [18] via the iterative procedure described in [19].

\section{Gamma-ray analysis}

\subsection{Data and analysis procedure}

In this work we used 12 years of Fermi-LAT data and the Instrument Response Functions (IRFs) P8R3_SOURCE_V2. We separated the events into four types depending on the quality of the direction reconstruction. We chose the energy thresholds for each event type to have a PSF $68 \%$ containment radius smaller than $0.7^{\circ}$. The overall energy range of the analysis is $0.5 \mathrm{TeV}$ to $1000 \mathrm{TeV}$. We also applied a selection on the zenith angle to reduce the contamination from the Earth's $\gamma$-ray emission.

We analysed $\gamma$-ray data in a region with Galactic longitude $73^{\circ} \leq l \leq 87^{\circ}$ and with Galactic latitude $|b| \leq 15^{\circ}$. We used Fermipy (v1.0.1) and the fermitools (v2.0.8). We performed a binned likelihood analysis based on Poisson statistics. We chose a spatial binning of $1 / 10^{\circ}$ and a spectral binning of 10 energy bins per decade. 

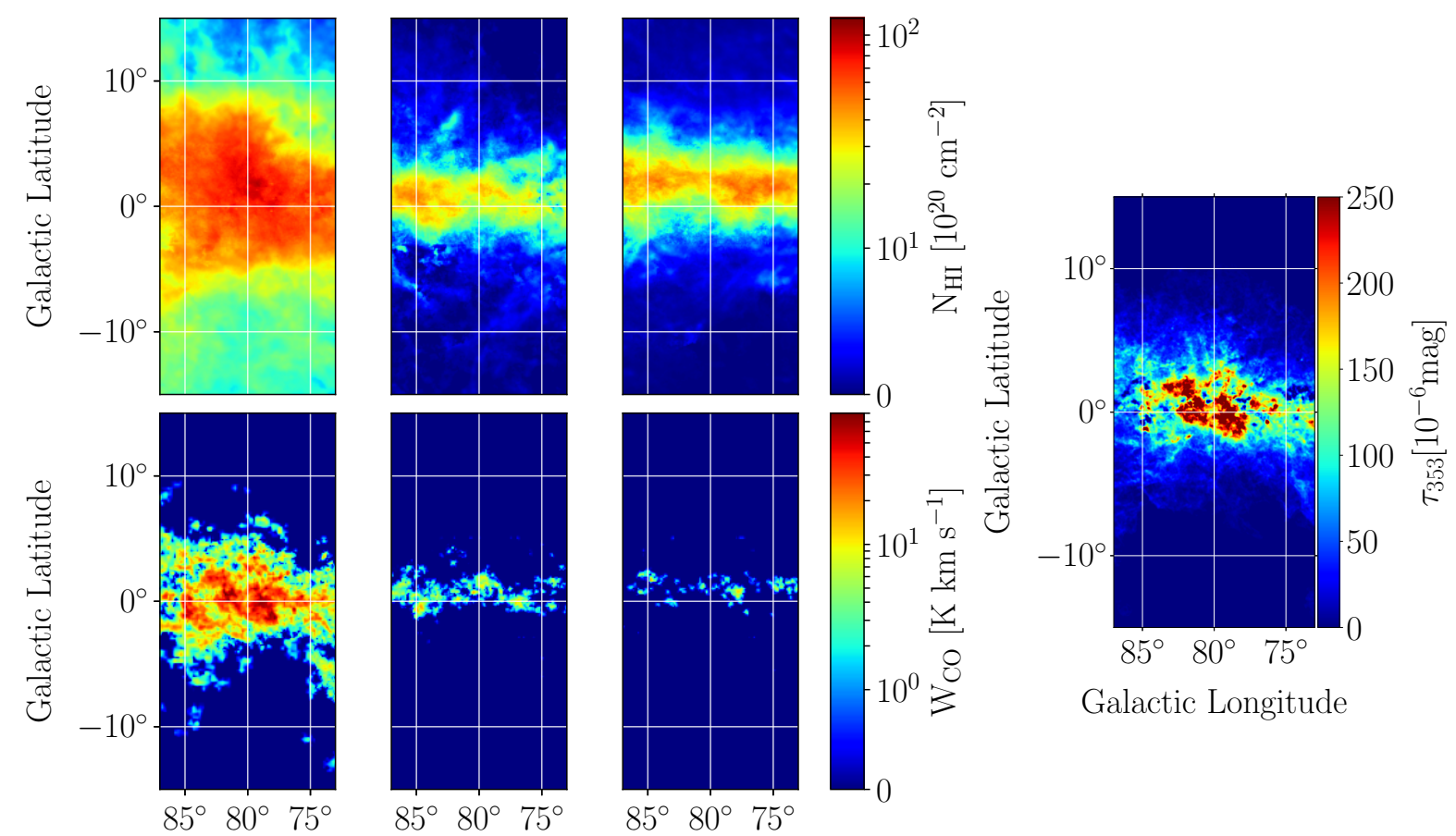

Galactic Longitude

Galactic Longitude

Figure 2: H i column densities (top) and $W_{C O}$ intensities (bottom) for (left to right): Local Arm, Perseus Arm, and Outer Arm and beyond. The separated figure on the right is the final DNM map.

We supposed that the variation of large-scale CR densities are small within each of the structures separated along the line of sight (see Sect. 2). Therefore, we can model large-scale interstellar $\gamma$-ray emission from gas as a linear combination of the gas column densities in the different phases and in the different structures along the line of sight. The linear combination coefficients correspond to emissivities, respectively $\gamma$-ray emissivity per hydrogen atom $\left(q_{\mathrm{H}_{I}}\right)$, per $W_{C O}$ unit $\left(q_{C O}\right)$, and per $\tau_{353}$ unit $\left(q_{D N M}\right)$. We also added a model for inverse-Compton (IC) emission generated using GALPROP code (run SY_Z6_R30_T150_C2 [20]).In addition to the Galactic interstellar emission model, we included an isotropic component derived from a fit to the all-sky emission excluding the Galactic plane $\left(|b|<10^{\circ}\right)$ and regions around celestial poles $\left(|b|>60^{\circ}\right)[21]$.

The model is completed with sources from the fourth Fermi-LAT gamma-ray sources catalogue, data release 2 [22]. We replaced the model for the $\gamma$-Cygni SNR (4FGL J2021.0+4031e) by two components, a shell and a northern component [23]. We also replaced the two sources associated with the Cygnus Loop, namely 4FGL J2051.0+3049e and 4FGL J2056.4+3142 by two templates based on X-ray and UV emission from the remnant [24].

\subsection{Morphological analysis}

We considered different models for the morphology of the cocoon:

- a 2D Gaussian model as in [2]; 
- a $6^{\circ} \times 6^{\circ} 8 \mu \mathrm{m}$ intensity map centered on $l=79.5^{\circ}$ and $b=1^{\circ}$ from MSX (Midcourse Space Experiment) [25], to trace the borders of the ionized cavities carved by stellar activity in Cygnus X;

- a free-free intensity map obtained from Planck data [26], selected within a region determined based on the contours of the $8 \mu \mathrm{m}$ map to trace the interior of the ionized cavities carved by stellar activity in Cygnus X;

- a combination of the two aforementioned maps with a 2D Gaussian, which was suggested by visual inspection of the residuals.

The results are shown in Table 1. The model which includes the free-free template and the Gaussian is favored by the largest likelihood value, which corresponds to a very significant improvement with respect to the Gaussian alone. When including the $8 \mu \mathrm{m}$ or free-free templates the extension of the Gaussian becomes larger. For the extension of the 2D Gaussian, we report a significant increase of the extension when the new components are in the model. Even if we use only the 2D Gaussian, its extension is bigger than the previous value of $3.0^{\circ} \pm 0.3^{\circ}$ [2]. The position of the Gaussian remains consistent across the different models.

\begin{tabular}{ccccc}
\hline Model & log-likelihood & \multicolumn{3}{c}{ Gaussian's parameters } \\
& & R.A. & Dec. & $r_{68 \%}$ \\
\hline 2D Gaussian & 0 & $79.47^{\circ} \pm 0.25^{\circ}$ & $1.4^{\circ} \pm 0.25^{\circ}$ & $3.61^{\circ} \pm 0.03^{\circ}$ \\
$8 \mu \mathrm{m}$ & -416 & - & - & - \\
free-free & -845 & - & - & - \\
$8 \mu \mathrm{m}+$ 2D Gaussian & 274 & $79.86^{\circ} \pm 0.13^{\circ}$ & $1.1^{\circ} \pm 0.13^{\circ}$ & $5.2^{\circ} \pm 0.1^{\circ}$ \\
free-free + 2D Gaussian & 345 & $79.54^{\circ} \pm 0.1^{\circ}$ & $1.22^{\circ} \pm 0.1^{\circ}$ & $4.51^{\circ} \pm 0.08^{\circ}$ \\
\hline
\end{tabular}

Table 1: [PRELIMINARY] Results of the morphological analysis. The log-likelihood values are given with respect to the 2D Gaussian model.

The residuals from the three best morphological models are shown in Figure 3. We can see that including the $8 \mu \mathrm{m}$ or free-free templates results is a sizeable improvement around the cocoon. However there still remain residuals that deserve further investigation.

\subsection{Spectral analysis}

Figure 4 shows the spectral energy distribution of the cocoon for the best-fit model. the spectrum of the Gaussian component is harder and brighter than in the original analysis [2], but close to results in the 4FGL-DR2 catalog. The spectra of the Gaussian and free-free components are different, and the latter is closer to the results in [2]. Our spectrum extends to $1 \mathrm{TeV}$ and enables us to compare with the spectra derived by ground-based air-shower detectors. The spectrum measured by ARGO in the few-hundred GeV energy range is not compatible with the total emission we measure, but it is consistent with the spectrum of the free-free component. If we assume that HAWC observes the total emission captured by the LAT, our results strengthen the evidence for a steepening of the spectrum around $1 \mathrm{TeV}$. 

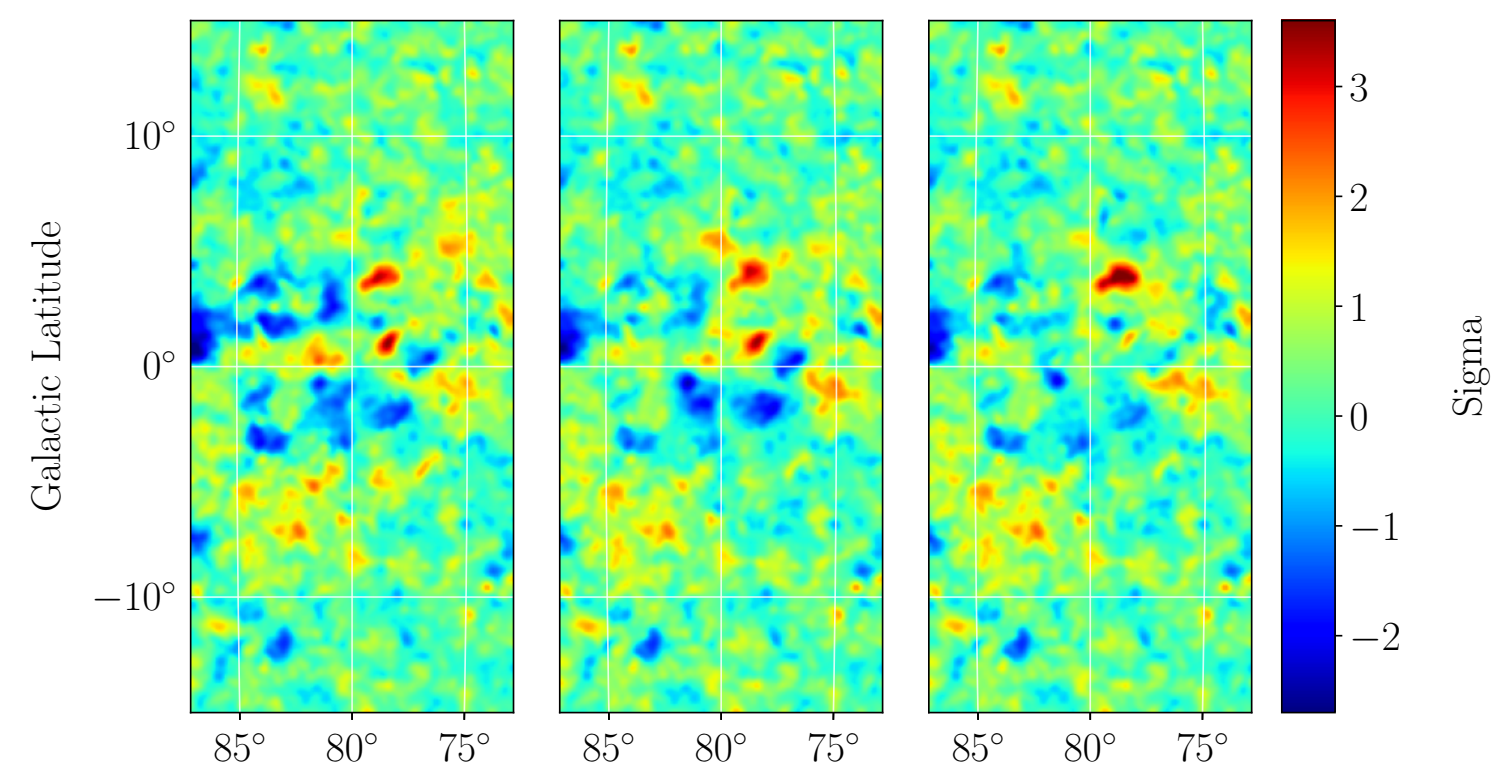

Galactic Longitude

Figure 3: [PRELIMINARY] Residuals in sigma values. From left to right, we have the residuals of the Gaussian only model, the Gaussian $+8-\mu$ m model and the Gaussian + free-free model.

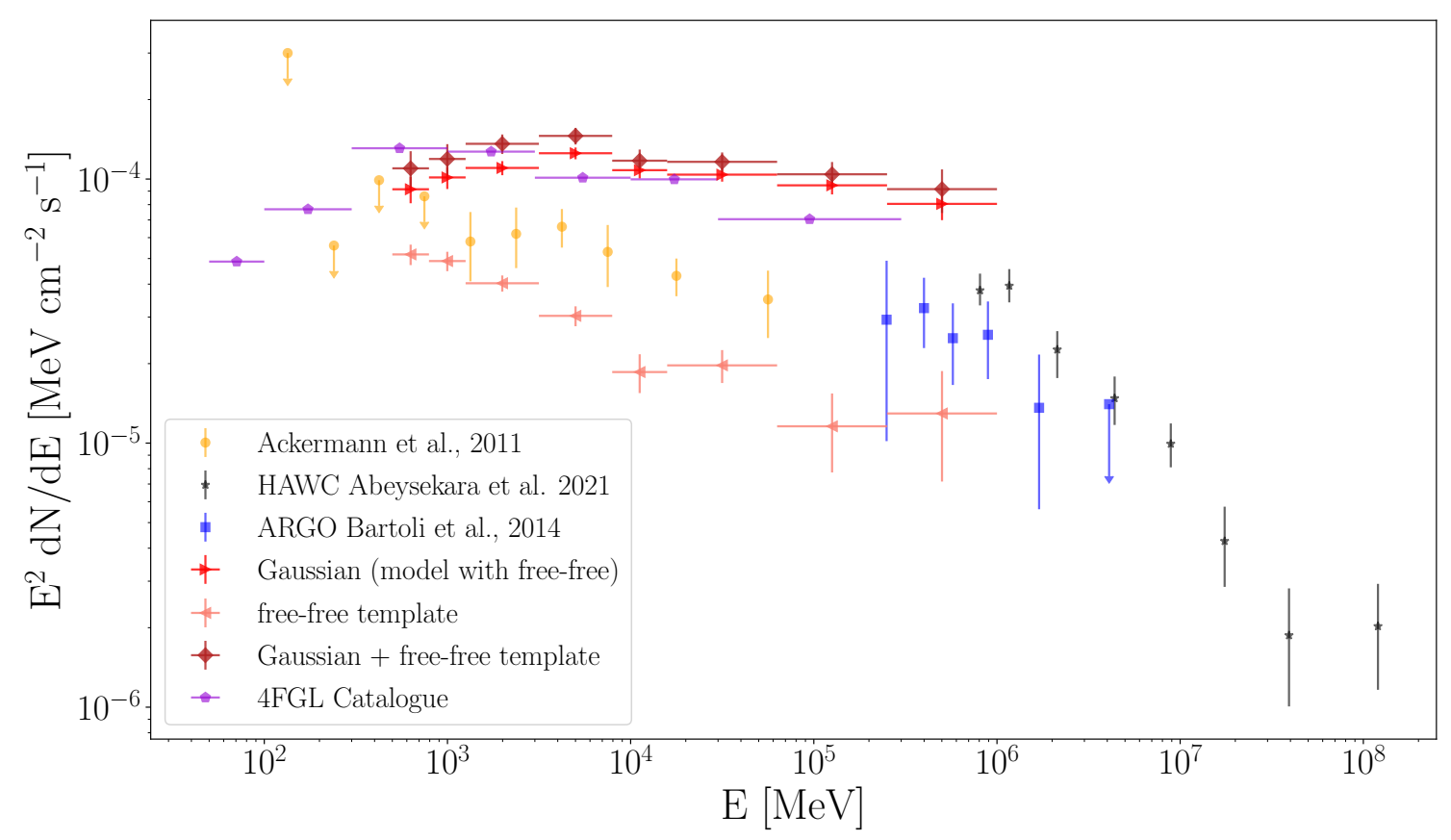

Figure 4: [PRELIMINARY] Spectral energy distribution of the cocoon from the LAT and ground-based observations. In red we show the two components of the best-fit model (Gaussian and free-free template) and their sum (the uncertainty does not take into account the covariance of the two components). 


\section{Final remarks}

Our preliminary results suggest that gamma-ray emission from the Cygnus cocoon has a complex morphology with multiple components which show different spectral properties. The best-fit model obtained so far includes a free-free template that captures a central excess coincident with the ionized cavities carved by the MSFRs in the interstellar medium, plus a Gaussian with larger extension than in earlier analyses [2].

The residuals for the best-fit model are still not completely satisfactory, therefore the model may still be improved. Once a satisfactory model is obtained, we will use it to determine how the morphology of the cocoon evolves with energy and how the spectrum varies in different regions, and explore the implications for the origin and transport of the freshly accelerated particles.

The Fermi-LAT Collaboration acknowledges support for LAT development, operation and data analysis from NASA and DOE (United States), CEA/Irfu and IN2P3/CNRS (France), ASI and INFN (Italy), MEXT, KEK, and JAXA (Japan), and the K.A. Wallenberg Foundation, the Swedish Research Council and the National Space Board (Sweden). Science analysis support in the operations phase from INAF (Italy) and CNES (France) is also gratefully acknowledged. This work performed in part under DOE Contract DE-AC02-76SF00515.

\section{References}

[1] W.R. Binns, M.E. Wiedenbeck, M. Arnould, A.C. Cummings, J.S. George, S. Goriely et al., Astrophysical Journal 634 (2005) 351 [astro-ph/0508398].

[2] M. Ackermann, M. Ajello, A. Allafort, L. Baldini, J. Ballet, G. Barbiellini et al., Science 334 (2011) 1103.

[3] A.U. Abeysekara, A. Albert, R. Alfaro, C. Alvarez, J.R.A. Camacho, J.C. Arteaga-Velázquez et al., Nature Astronomy (2021) [2103.06820].

[4] F. Aharonian, A.G. Akhperjanian, A.R. Bazer-Bachi, M. Beilicke, W. Benbow, D. Berge et al., Astronomy and Astrophysics 467 (2007) 1075 [astro-ph/0703427].

[5] S. Ohm, J.A. Hinton and R. White, Monthly Notices of the Royal Astronomical Society 434 (2013) 2289 [1306. 5642].

[6] HESS Collaboration, A. Abramowski, F. Aharonian, F.A. Benkhali, A.G. Akhperjanian, E.O. Angüner et al., Nature 531 (2016) 476 [1603.07730].

[7] R.-z. Yang and F. Aharonian, Astronomy and Astrophysics 600 (2017) A107 [1612 . 02250].

[8] J. Katsuta, Y. Uchiyama and S. Funk, Astrophysical Journal 839 (2017) 129 [1704.06110].

[9] X.-N. Sun, R.-Z. Yang, Y.-F. Liang, F.-K. Peng, H.-M. Zhang, X.-Y. Wang et al., Astronomy and Astrophysics 639 (2020) A80 [2006.00879].

[10] G. Maurin, A. Marcowith, N. Komin, F. Krayzel and G. Lamanna, Astronomy and Astrophysics 591 (2016) A71 [1605.04202]. 
[11] T. Joubaud, I.A. Grenier, J.M. Casandjian, T. Tolksdorf and R. Schlickeiser, Astronomy and Astrophysics 635 (2020) A96 [2001. 10139].

[12] HI4PI Collaboration, N. Ben Bekhti, L. Flöer, R. Keller, J. Kerp, D. Lenz et al., Astronomy and Astrophysics 594 (2016) A116 [1610.06175].

[13] A.R. Taylor, S.J. Gibson, M. Peracaula, P.G. Martin, T.L. Landecker, C.M. Brunt et al., Astronomical Journal 125 (2003) 3145.

[14] M. Ackermann, M. Ajello, A. Allafort, L. Baldini, J. Ballet, G. Barbiellini et al., Astronomy and Astrophysics 538 (2012) A71 [1110.6123].

[15] T.M. Dame, D. Hartmann and P. Thaddeus, The Astrophysical Journal 547 (2001) 792 [astro-ph/0009217].

[16] Planck Collaboration, Fermi Collaboration, P.A.R. Ade, N. Aghanim, G. Aniano, M. Arnaud et al., Astronomy and Astrophysics 582 (2015) A31 [1409. 3268].

[17] I.A. Grenier, J.-M. Casandjian and R. Terrier, Science 307 (2005) 1292 [https://science.sciencemag.org/content/307/5713/1292.full.pdf].

[18] Planck Collaboration, Aghanim, N., Ashdown, M., Aumont, J., Baccigalupi, C., Ballardini, M. et al., Astronomy and Astrophysics 596 (2016) A109.

[19] L. Tibaldo, S.W. Digel, J.M. Casandjian, A. Franckowiak, I.A. Grenier, G. Jóhannesson et al., Astrophysical Journal 807 (2015) 161 [1505.04223].

[20] M. Ackermann, M. Ajello, W.B. Atwood, L. Baldini, J. Ballet, G. Barbiellini et al., Astrophysical Journal 750 (2012) 3 [1202 . 4039].

[21] S. Abdollahi, F. Acero, M. Ackermann, M. Ajello, W.B. Atwood, M. Axelsson et al., Astrophysical Journal Supplement Series 247 (2020) 33 [1902 . 10045].

[22] J. Ballet, T.H. Burnett, S.W. Digel and B. Lott, arXiv e-prints (2020) arXiv:2005.11208 [2005.11208].

[23] MAGIC Collaboration, V.A. Acciari, S. Ansoldi, L.A. Antonelli, A. Arbet Engels, D. Baack et al., arXiv e-prints (2020) arXiv:2010.15854 [2010 .15854].

[24] A. Tutone, J. Ballet, F. Acero, A. D’Aì and G. Cusumano, In preparation (2021) .

[25] M.P. Egan, R.F. Shipman, S.D. Price, S.J. Carey, F.O. Clark and M. Cohen, The Astrophysical Journal 494 (1998) L199.

[26] Planck Collaboration, R. Adam, P.A.R. Ade, N. Aghanim, M.I.R. Alves, M. Arnaud et al., Astronomy and Astrophysics 594 (2016) A10 [1502.01588]. 